\title{
Influence of Autonomic Nervous Dysfunction Characterizing Effect of Diabetes Mellitus on Heart Rate Response and Exercise Capacity in Patients Undergoing Cardiac Rehabilitation for Acute Myocardial Infarction
}

\author{
Yusuke Kasahara, MS* ${ }^{*}$; Kazuhiro Izawa, $\mathrm{PhD}^{* *}$; Kazuto Omiya, $\mathrm{MD}^{\dagger}$; \\ Naohiko Osada, MD ${ }^{\dagger}$; Satoshi Watanabe, BS**; Masakazu Saitoh, $\mathrm{MS}^{\dagger \dagger}$; \\ Atsuhiko Matsunaga, $\mathrm{PhD}$; Takashi Masuda, MD*
}

\begin{abstract}
Background The aim of this study was to clarify the influence of sympathetic and parasympathetic nerve (SN and PN) dysfunction on the heart rate (HR) response to exercise and the exercise capacity of patients with acute myocardial infarction (AMI) and diabetes mellitus (DM).

Methods and Results Fifty-two male patients who underwent cardiopulmonary exercise testing (CPX) 1 month after onset of AMI were divided into 2 groups: $(\mathrm{DM}(+)$ group, $n=20$; DM $(-)$ group, $n=32)$. HR, peak oxygen uptake ( $\left.\dot{\mathrm{V}} \mathrm{O}_{2 \text { peak}}\right)$, and plasma norepinephrine (NE) levels were measured during CPX. The high-frequency power (HF) was analyzed by HR variability. The $\Delta \mathrm{HR} / \log \Delta \mathrm{NE}$ obtained from changes of $\mathrm{HR}$ and NE from rest to peak exercise and HR change from baseline to the minimum HF ( $\triangle \mathrm{HRHF}$ ) were calculated as parameters of HR response derived from SN and PN activities, respectively. $\Delta \mathrm{HR}$, $\dot{\mathrm{V}}_{2 \text { peak, }} \Delta \mathrm{HR} / \log \Delta \mathrm{NE}$, and $\Delta \mathrm{HRHF}$ were significantly lower in the $\mathrm{DM}(+)$ group than in the $\mathrm{DM}(-)$ group, and both of them showed positive correlations with $\dot{\mathrm{V}}$ 2peak.
\end{abstract}

Conclusion An inadequate HR response to exercise is a major factor causing a decline of exercise capacity, which is derived from both of SN and PN dysfunction, in AMI patients with DM. (Circ J 2006; 70: 1017-1025)

Key Words: Acute myocardial infarction; Autonomic nervous system; Diabetes mellitus; Exercise; Rehabilitation

$\mathbf{T}$ ype 2 diabetes mellitus (DM) is not only an independent risk factor for the development of coronary artery disease, but also contributes to mortality through the induction of arrhythmias or sudden death after acute myocardial infarction (AMI)! Furthermore, recent epidemiological surveys have shown that the morbidity of DM has rapidly increased in Japan in the past decade and in consequence the incidence of AMI complicated by DM has also increased remarkably? Because exercise capacity is reduced after AMI in patients with DM in comparison with similar patients without DM, comprehensive cardiac rehabilitation (CR) should be instituted in these patients after the onset of AMI, It is important to clarify the mechanisms underlying the decline of exercise capacity after AMI in patients with DM, in order to offer the appropriate exercise training program to them.

Heart rate (HR) response to exercise is regulated by the

(Received October 31, 2005; revised manuscript received May 19, 2006; accepted May 25, 2006)

*Department of Rehabilitation Medicine, St Marianna University School of Medicine Yokohama City Seibu Hospital, Yokohama, **Department of Rehabilitation Medicine, St Marianna University School of Medicine, Division of Cardiology, St Marianna University School of Medicine, Kawasaki, ${ }^{\dagger}$ Graduate School of Medical Science and $\$$ Department of Rehabilitation, School of Allied Health Sciences, Kitasato University, Sagamihara, Japan

Mailing address: Takashi Masuda, MD, Department of Rehabilitation, School of Allied Health Sciences, Kitasato University, 1-15-1 Kitasato, Sagamihara 228-8555, Japan. E-mail: tak9999@ med.kitasato-u.ac.jp balance between sympathetic and parasympathetic nerve ( $\mathrm{SN}$ and PN) activities in the human body5 An insufficient HR response to exercise caused by autonomic nervous dysfunction seems to be an important mechanisms in patients with DM who show decreased exercise capacity3,6 It is well known that norepinephrine (NE) secretion from the terminals of the SNs decreases during exercise in patients with $\mathrm{DM}$, as compared with normal subjects? A previous study concerning the relationship between HR response and SN activity during exercise showed that the HR response corresponding to NE secretion is reduced in AMI patients with DM, resulting in a decline in exercise capacity? In addition, it has been reported that patients with DM have not only SN dysfunction but also PN dysfunction, which is shown by a decrease in the high-frequency power (HF) in HR variability (HRV)? However, there are few reports evaluating the influence of PN activity separately from SN activity on the HR response to exercise and exercise capacity of patients with AMI, and moreover, the impact of DM in causing insufficient HR response to exercise. It is very important to clarify the mechanism of HR response to exercise regulated by $\mathrm{SN}$ and $\mathrm{PN}$ activities in order to prescribe appropriate CR that takes in to account the decreased exercise capacity in AMI patients with DM.

It is reported that an imbalance of autonomic nervous activities causes excessive elevation of blood pressure and $\mathrm{HR}$ during exercise training in patients with $\mathrm{AMI}^{9}$ and that the marked activation of SN induces ventricular arrhythmias in patients with coronary artery disease $!^{10}$ Thus, it is 


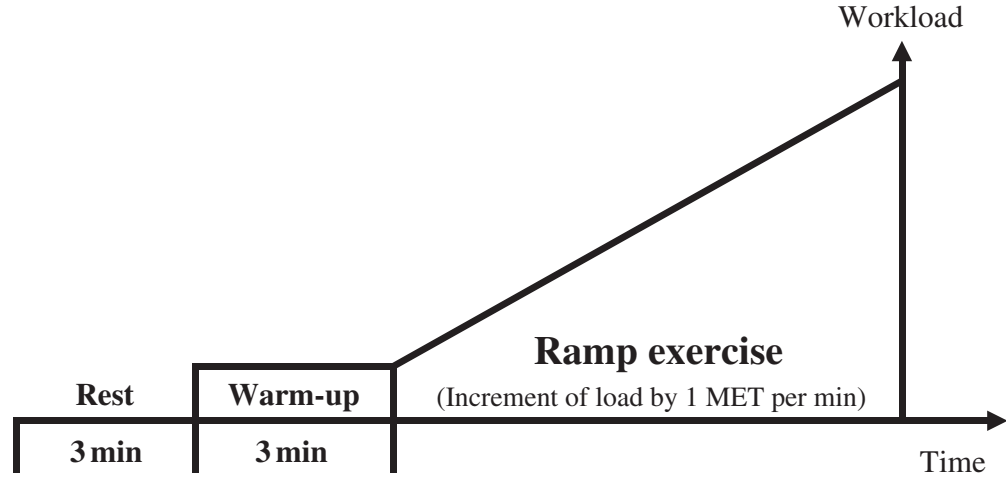

Fig 1. Protocol of cardiopulmonary exercise testing. MET, metabolic equivalent. important to assess the HR response derived from the balance between $\mathrm{SN}$ and PN activities, so that exercise training can be performed at a safe and effective intensity, and to avoid the risk of sudden cardiac death during exercise. Generally, the anaerobic threshold (AT) is used to decide the appropriate intensity of exercise training in $\mathrm{CR}, 1$ because neither elevation of the lactic acid level nor activation of the SN system occur at exercise intensities lower than the AT ${ }^{12,13}$ It is also known that the increase in HR in healthy subjects is predominantly derived from the decreased PN activity that occurs at an exercise intensity below the AT! ${ }^{13}$ However, the relationship between HR response to exercise and PN activity at intensities below the AT in AMI patients with DM remains unclear.

The purpose of the present study was to clarify the influence of the autonomic nervous dysfunction characterizing $\mathrm{DM}$ on exercise capacity in patients undergoing CR for AMI, focusing on separate $\mathrm{SN}$ and $\mathrm{PN}$ activities that affected HR response to exercise.

\section{Methods}

\section{Patients}

Male patients who were admitted to St Marianna University School of Medicine Hospital from November 1998 to March 2000 because of AMI were eligible for the present study, if they completed a routine 4 -week CR program while hospitalized and performed cardiopulmonary exercise testing (CPX) 1 month after onset of AMI. Patients who were older than 70 years, had severe heart failure (New York Heart Association class 4), uncontrolled arrhythmia, atrial fibrillation, or left bundle branch block, or who underwent coronary artery bypass grafting surgery or treatment with $\beta$-blockers were excluded. Female patients were also excluded because it is well known that they have significantly lower exercise capacity than males ${ }^{14}$ Consequently, 52 male patients were recruited for the study and were divided into 2 groups according to whether or not they had DM: there were 20 patients in the DM(+) group and 32 in the DM(-) group.

The diagnosis of AMI was confirmed by the existence of chest pain that lasted $30 \mathrm{~min}$ or more, ST-segment elevation of $0.1 \mathrm{mV}$ or more in at least 2 contiguous leads of 12lead electrocardiogram (ECG), and a significant increase of serum creatine kinase-myocardial band (CK-MB) levels 2-fold or more from the upper limit of the normal range. DM was diagnosed if patients were under treatment with oral hypoglycemic agents or insulin injection, or if an oral glucose tolerance test performed after admission was positive. In the $\mathrm{DM}(+)$ group, 18 patients were being treated with oral hypoglycemic agents, and 7 by insulin injection.

Age, body weight, height, body mass index, location of AMI, maximum CK-MB (max CK-MB) and glycosylated hemoglobin $(\mathrm{HbA} 1 \mathrm{c})$ were recorded as clinical characteristics in all patients. While the patients were hospitalized for AMI, they underwent coronary angiography (CAG) and left ventriculography (LVG). The number of significant stenoses of the coronary arteries ( $\geq 75 \%$; especially left main trunk, $\geq 50 \%$ ) was evaluated based on the definition that coronary stenosis was measured as the luminal diameter loss in comparison with the nearest proximal normal segment. Left ventricular ejection fraction (LVEF) was calculated via biplane LVG. All patients underwent direct percutaneous transluminal coronary angioplasty for AMI on admission. The medications, including nitrates, calcium channel antagonists, and angiotensin-converting enzyme inhibitors were recorded, when CPX was performed.

The study was approved by the St Marianna University School of Medicine institutional committee on human research. Informed consent was given by each patient before entering the study 1 month after onset of AMI.

\section{Exercise Testing}

Symptom-limited CPX was performed with a treadmill (MAT-2500, Fukuda Denshi, Tokyo, Japan) according to a ramp protocol. After patients rested for $3 \mathrm{~min}$ on the treadmill, exercise was performed with the increment of load by $1 \mathrm{MET} / \mathrm{min}$ following a 3-min warm-up period (speed, $1.0 \mathrm{mile} / \mathrm{h}$; slope, 0\%) (Fig 1). HR and 12-lead ECG were continuously monitored by the Stress Test System (ML5000, Fukuda Denshi), and systolic and diastolic blood pressure (SBP and DBP) were measured by the cuff method via an automatic blood pressure monitor (STBP780, Colin, Aichi, Japan) at 1-min intervals throughout the CPX period. As expired gas was sampled using a breathby-breath method, oxygen uptake $\left(\dot{\mathrm{VO}}_{2}\right)$, carbon dioxide production $\left(\dot{\mathrm{VCO}}_{2}\right)$, and tidal volume $(\dot{\mathrm{V} E})$ were measured simultaneously with a respiromonitor (AE-280, Minato Ikagaku, Tokyo, Japan) and a gas analyzer (MG-360, Minato Ikagaku) during CPX. The endpoint of CPX was determined according to the criteria of the American College of Sports Medicine ${ }^{15}$ or by a leveling off of $\dot{\mathrm{VO}}_{2}$ that showed no change despite increasing exercise intensity. None of the patients showed ischemic ECG changes during CPX.

The HR at rest (HRrest), warm-up (HRwu), and peak exercise (HRpeak) were determined from the HR recorded throughout the CPX period, and the increase from HRrest to $H_{R}$ peak $(\triangle H R)$ was calculated. The duration from the beginning of exercise to peak exercise (exercise time) was mea- 


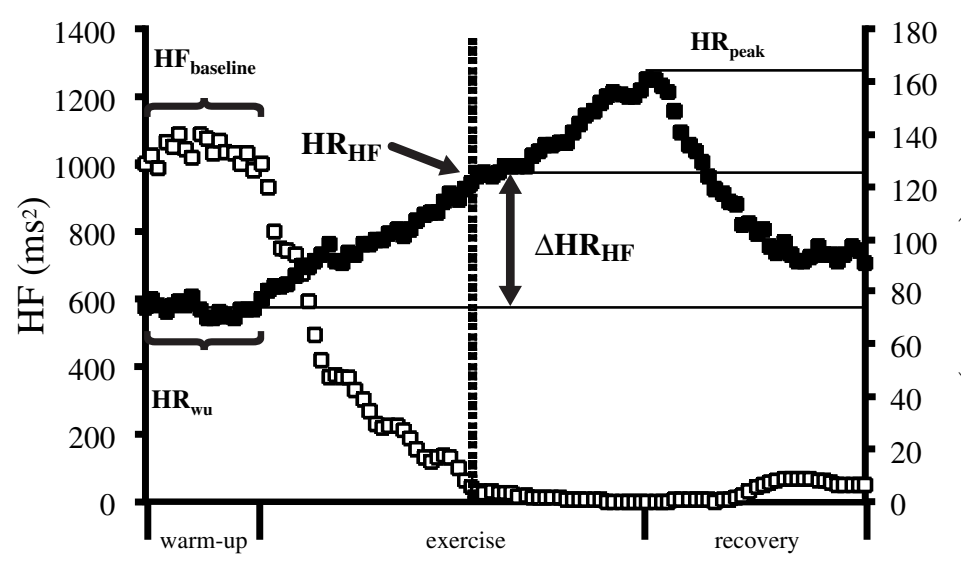

Fig 2. Time course in heart rate (HR) and highfrequency power (HF) of heart rate variability during cardiopulmonary exercise testing. HFbaseline, average of high-frequency power at warm-up period; $\mathrm{HRHF}$, the HR when HF reached the minimum level; $\Delta \mathrm{HRHF}$, HR change from HRwu to HRHF; HRpeak, heart rate at peak exercise. sured, and HR slope indicating an increase of HR per min was also calculated throughout the CPX period. In addition, the time constant of HR decay for the first 30 s of recovery period (T30) was calculated by the method described by Imai et al ${ }^{16}$ HR reserve (HRR) estimated according to patient's age and HRrest, and the \%HRR were calculated: ${ }^{17}$

$$
\begin{aligned}
& \mathrm{HRR}=220-\text { age }- \text { HRrest } \\
& \% \mathrm{HRR}=(\text { HR peak }- \text { HRrest }) /(220-\text { age }- \text { HRrest }) \times 100 \%
\end{aligned}
$$

The gas exchange ratio ( $\dot{\mathrm{V}} \mathrm{CO}_{2} / \dot{\mathrm{V}}_{2}$ : GER) was calculated, and the $\dot{\mathrm{V}}_{2}$ slope was assessed as an increase of $\dot{\mathrm{V}}_{2}$ per min. The oxygen pulse $\left(\mathrm{O}_{2}\right.$ pulse $)$ indicating stroke volume was calculated: ${ }^{18}$

$$
\mathrm{O}_{2} \text { pulse }=\dot{\mathrm{V}} \mathrm{O}_{2} / \mathrm{HR}
$$

We measured SBP, $\dot{V}_{2}$, GER and $\mathrm{O}_{2}$ pulse at rest (SBPrest, $\dot{\mathrm{V}} \mathrm{O}_{2 \text { rest, }}$ GRErest and $\mathrm{O}_{2}$ pulserest, respectively), and $\mathrm{SBP}, \dot{\mathrm{V}} 2$, GER and $\mathrm{O}_{2}$ pulse at peak exercise (SBPpeak, $\dot{\mathrm{V}} \mathrm{O}_{2 \text { peak, }}$ GREpeak and $\mathrm{O}_{2}$ pulsepeak, respectively), and calculated the change from $\mathrm{O}_{2}$ pulserest to $\mathrm{O}_{2}$ pulsepeak $\left(\Delta \mathrm{O}_{2}\right.$ pulse). After determining the AT by the V-slope method, ${ }^{11}$ we measured $\mathrm{HR}, \mathrm{SBP}, \mathrm{VO}_{2}$, GER and $\mathrm{O}_{2}$ pulse at the AT (HRAt, SBPat, VंOzat, GREAT and $\mathrm{O}_{2}$ pulseat, respectively). Then, we calculated the ratio of HRAT to HRpeak (HRAT/HRpeak $\times 100$ ), to assess the workload at HRAT relative to that at peak exercise.

\section{Blood Sampling}

Blood samples for measurement of NE were obtained from a catheter inserted into an antecubital vein before warm-up and at peak exercise, and were immediately icecooled. After centrifugation at $3,000 \mathrm{rpm}$ at $4^{\circ} \mathrm{C}$ for $10 \mathrm{~min}$, separated plasma was stored at $-80^{\circ} \mathrm{C}$ until analyzed. The plasma concentration of NE was determined by using high performance liquid chromatography.

The change from NE at rest (NEbaseline) to NE at peak exercise (NEpeak) was converted to a logarithmic value $(\log \Delta \mathrm{NE})$, because NE increases exponentially during incremental exercise 19 Consequently, sympathetic nervous activity was assessed as the ratio of $\Delta \mathrm{HR}$ to $\log \Delta \mathrm{NE}$ $(\Delta \mathrm{HR} / \log \Delta \mathrm{NE})$.

\section{$H R V$}

R-R intervals obtained from ECG monitoring throughout CPX were converted to digital data by $250 \mathrm{~Hz}$ sampling rate for HRV analysis. The HRV of the R-R intervals was analyzed by the MemCalc method ${ }^{20}$ and the frequency domain power spectra were calculated for the low $(0.04-$
Table 1 Parameters Indicating Autonomic Nervous Activity

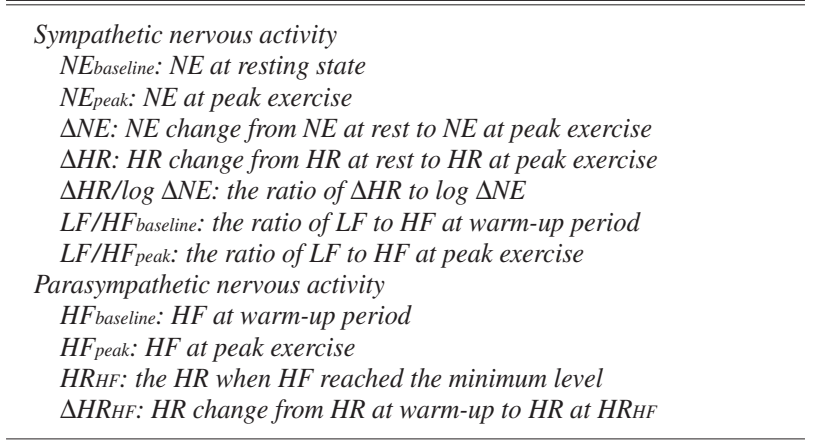

$N E$, norepinephrine; $\triangle N E$, change of $N E$ concentration from rest to peak exercise; $\triangle H R$, change of heart rate $(H R)$ from rest to peak exercise; $L F$, low-frequency power in heart rate variability; HF, high-frequency power in heart rate variability; $\triangle H R H F, H R$ change from $H R$ at warm-up to $H R$ at the mini-mum level during cardiopulmonary exercise testing.

$0.15 \mathrm{~Hz})$ and high $(0.15-0.4 \mathrm{~Hz})$ frequency components of the power spectrum (low-frequency power (LF) and HF, respectively). The power spectrum of the HF component is known to reflect cardiac PN activity, and the ratio of LF to $\mathrm{HF}(\mathrm{LF} / \mathrm{HF})$ indicates the predominance of cardiac SN activity. $\mathrm{LF}, \mathrm{HF}$, and $\mathrm{LF} / \mathrm{HF}$ in the time series were analyzed at 10-s intervals during CPX. The averages of LF, HF and $\mathrm{LF} / \mathrm{HF}$ were calculated as baseline values using all of the 18 data during warm-up period (LFbaseline, HFbaseline and $\mathrm{LF} / \mathrm{HF}$ baseline, respectively). They were calculated as the moving average using consecutive data of LF, HF and LF/HF, respectively, during ramp exercise. Furthermore, the averages of LF, HF and LF/HF at HRpeak were calculated from 2 data before the end of ramp exercise (LFpeak, $\mathrm{HF}_{\text {peak }}$ and $\mathrm{LF} / \mathrm{HF}$ peak, respectively), to avoid the interference of data from a recovery period. After $\Delta \mathrm{HR}$ was divided into 5 stages by the $20 \%$ of $\Delta \mathrm{HR}$ in the ramp exercise, LF, HF and LF/HF at HR of each stage (HRrest + $20 \% \Delta \mathrm{HR}$, HRrest $+40 \% \Delta \mathrm{HR}$, HRrest $+60 \% \Delta \mathrm{HR}$, HRrest + $80 \% \Delta \mathrm{HR}$ and HRpeak) were assessed in the 2 groups to compare the changes of $\mathrm{SN}$ and $\mathrm{PN}$ activities to the relative exercise intensity during exercise. Because arrhythmias such as premature ventricular contraction (PVC) and premature atrial contraction (PAC) affect HRV analysis, we excluded patients who had more than 4 PVCs or PACs per min during exercise. The number of arrhythmia episodes was automatically counted by a diagnosis program during the 12-lead ECG study. 
Table 2 Patients' Characteristics

\begin{tabular}{|c|c|c|c|}
\hline & $\begin{array}{c}\text { DM (-) } \\
\text { group }\end{array}$ & $\begin{array}{c}\text { DM (+) } \\
\text { group }\end{array}$ & $p$ value \\
\hline Number & 32 & 20 & \\
\hline Age (years) & $57.0 \pm 1.5$ & $58.2 \pm 1.7$ & $N S$ \\
\hline Body weight $(\mathrm{kg})$ & $62.5 \pm 1.3$ & $61.4 \pm 1.8$ & $N S$ \\
\hline Height $(\mathrm{cm})$ & $163.5 \pm 1.0$ & $164.3 \pm 1.4$ & $N S$ \\
\hline$B M I\left(k g / m^{2}\right)$ & $23.4 \pm 0.5$ & $22.8 \pm 0.6$ & $N S$ \\
\hline $\operatorname{Max} C K-M B(I U / L)$ & $233.7 \pm 44.2$ & $248.2 \pm 44.2$ & $N S$ \\
\hline$H b A l c(\%)$ & $5.3 \pm 0.2$ & $7.2 \pm 0.2$ & $<0.001$ \\
\hline $\operatorname{LVEF}(\%)$ & $50.7 \pm 1.7$ & $50.4 \pm 4.3$ & $N S$ \\
\hline$L V E F<40 \%$ & $7(22 \%)$ & $5(25 \%)$ & $N S$ \\
\hline \multicolumn{4}{|l|}{ Location of AMI } \\
\hline Anterior wall & $9(28.1 \%)$ & $4(20.0 \%)$ & $N S$ \\
\hline Inferior wall & $16(50.0 \%)$ & $13(65.0 \%)$ & $N S$ \\
\hline Lateral or posterior wall & $7(21.9 \%)$ & $3(20.0 \%)$ & $N S$ \\
\hline \multicolumn{4}{|l|}{ Medication } \\
\hline Nitrates & $26(81.3 \%)$ & $15(75 \%)$ & $N S$ \\
\hline Ca antagonist & $7(21.9 \%)$ & $5(25 \%)$ & $N S$ \\
\hline ACE inhibitor & $23(72.9 \%)$ & $13(65.0 \%)$ & $N S$ \\
\hline Oral hypoglycemic agent & - & $18(90.0 \%)$ & - \\
\hline Insulin & - & $7(35.0 \%)$ & - \\
\hline \multicolumn{4}{|c|}{ Significant stenosis of coronary artery before PCI } \\
\hline 1 vessel disease & $17(53.1 \%)$ & $10(50.0 \%)$ & $N S$ \\
\hline 2 vessel disease & $13(40.6 \%)$ & $8(40.0 \%)$ & $N S$ \\
\hline 3 vessel disease & $2(6.3 \%)$ & $2(10.0 \%)$ & $N S$ \\
\hline
\end{tabular}

Data are mean $\pm S E$.

$D M$, diabetes mellitus; BMI, body mass index; Max CK-MB, maximum creatine kinase-myocardial band; HbAlc, glycosylated hemoglobin; LVEF, left ventricular ejection fraction; AMI, acute myocardial infarction; ACE, angiotensin-converting enzyme; $P C I$, percutaneous coronary intervention.

Fig 2 shows the changes in HR and HF during CPX. HF decreased at the beginning of exercise and HR increased linearly with the incremental workload. HF converged on a lower value and then fixed without showing any change during exercise. When the HF reached the minimum level, the HR was defined as HRHF, indicating that PN activity had nearly disappeared during exercise ${ }^{21,22}$ The HR change from HRwu to HRHF was defined as $\Delta \mathrm{HRHF}$, indicating the suppression of PN activity. In the present study, HRHF was clearly shown in all patients. To assess the workload at HRHF relative to that at peak exercise, we calculated the ratio of HRHF to HRpeak (HRHF/HRpeak $\times 100)$. The parameters used in the present study, indicating the changes in SN and $\mathrm{PN}$ activities, are summarized in Table 1.

\section{Statistical Analysis}

All data are expressed as the mean \pm SE. An unpaired t-test or Mann-Whitney U-test was used to compare the DM (+) and DM (-) groups, and a paired t-test was used to compare HRAT and HRHF in each group. Pearson's correlation coefficient was used in a regression analysis to determine the correlations between parameters of cardiac function or autonomic nervous activities (LVEF, max CK-MB, NEbaseline, HFbaseline, $\Delta \mathrm{HR} / \log \Delta \mathrm{NE}$ or $\Delta \mathrm{HRHF}$ ) and peak $\dot{\mathrm{V}} 2$, and between $\Delta \mathrm{HR} / \log \Delta \mathrm{NE}$ and $\Delta \mathrm{HRHF}$. A two-way analysis of variance for repeated-measures (2 groups vs 6 stages: HRwu, $\mathrm{HR}_{\text {rest }}+20 \% \Delta \mathrm{HR}, \mathrm{HR}$ rest $+40 \% \Delta \mathrm{HR}, \mathrm{HR} r \mathrm{st}+60 \% \Delta \mathrm{HR}$, $\mathrm{HR}_{\text {rest }}+80 \% \Delta \mathrm{HR}$ and HReak) was used to analyze the differences in LF, HF and LF/HF among the 6 stages and between the $\mathrm{DM}(-)$ and $\mathrm{DM}(+)$ groups during CPX. If the F ratio was significant, Tukey's multiple range test was applied to find the significant differences between the adjacent 2 stages and between the DM(-) and DM(+) groups. Statistical analyses were performed with SPSS 12.0J statis-
Table 3 Parameters for Cardiopulmonary Exercise Testing

\begin{tabular}{|c|c|c|c|}
\hline & $\begin{array}{c}\text { DM (-) } \\
\text { group }\end{array}$ & $\begin{array}{c}\text { DM (+) } \\
\text { group }\end{array}$ & $p$ value \\
\hline HRrest (beats/min) & $71.2 \pm 1.7$ & $77.0 \pm 2.6$ & $N S$ \\
\hline SBP rest $(m m H g)$ & $122.3 \pm 3.5$ & $125.3 \pm 3.5$ & $N S$ \\
\hline$\dot{V} O_{2 r e s t}\left(\mathrm{ml} \cdot \mathrm{min}^{-1} \cdot \mathrm{kg}^{-1}\right)$ & $4.0 \pm 0.1$ & $4.2 \pm 0.2$ & $N S$ \\
\hline GER rest & $0.83 \pm 0.1$ & $0.83 \pm 0.1$ & $N S$ \\
\hline $\mathrm{O}_{2}$ pulserest $\left(\mathrm{ml} \cdot \mathrm{min}^{-1} \cdot\right.$ beat $\left.^{-1}\right)$ & $3.6 \pm 0.1$ & $3.3 \pm 0.1$ & $N S$ \\
\hline HRAT (beats/min) & $106.6 \pm 2.3$ & $104.2 \pm 2.4$ & NS \\
\hline SBPAT $(m m H g)$ & $147.3 \pm 3.7$ & $143.2 \pm 4.5$ & $N S$ \\
\hline$\dot{V} O_{2 A T}\left(\mathrm{ml} \cdot \mathrm{min}^{-1} \cdot \mathrm{kg}^{-1}\right)$ & $16.4 \pm 0.4$ & $15.2 \pm 0.6$ & NS \\
\hline GERAT & $0.9 \pm 0.0$ & $0.9 \pm 0.0$ & $N S$ \\
\hline $\mathrm{O}_{2} \mathrm{pulse}$ at $\left(\mathrm{ml} \cdot \mathrm{min}^{-1} \cdot\right.$ beat $\left.^{-1}\right)$ & $9.8 \pm 0.3$ & $9.0 \pm 0.4$ & NS \\
\hline Exercise time $(s)$ & $533.4 \pm 15.3$ & $519.0 \pm 33.1$ & $N S$ \\
\hline HRpeak (beats/min) & $148.0 \pm 2.5$ & $135.8 \pm 3.1$ & 0.004 \\
\hline$S B P_{\text {peak }}(\mathrm{mmHg})$ & $173.8 \pm 4.3$ & $167.0 \pm 5.8$ & NS \\
\hline$\dot{V} O_{2 p e a k}\left(\mathrm{ml} \cdot \mathrm{min}^{-1} \cdot \mathrm{kg}^{-1}\right)$ & $25.8 \pm 0.6$ & $22.5 \pm 0.6$ & 0.002 \\
\hline GERpeak & $1.17 \pm 0.01$ & $1.17 \pm 0.01$ & $N S$ \\
\hline $\mathrm{O}_{2 p u l s e p e a k}\left(\mathrm{ml} \cdot \mathrm{min}^{-1} \cdot\right.$ beat $\left.^{-1}\right)$ & $10.9 \pm 0.3$ & $10.2 \pm 0.4$ & $N S$ \\
\hline$\Delta H R($ beats/min) & $76.8 \pm 2.5$ & $58.8 \pm 2.3$ & $<0.001$ \\
\hline$\Delta O_{2}$ pulse $\left(\mathrm{ml} \cdot \mathrm{min}^{-1} \cdot \mathrm{beat}^{-1}\right)$ & $7.4 \pm 0.3$ & $6.9 \pm 0.4$ & $N S$ \\
\hline$\% H R R(\%)$ & $84.5 \pm 2.9$ & $70.5 \pm 3.1$ & 0.002 \\
\hline HR slope (beats/min ${ }^{2}$ ) & $8.7 \pm 0.3$ & $7.5 \pm 0.4$ & 0.016 \\
\hline$\dot{\mathrm{V}} \mathrm{O}_{2}$ slope $\left(\mathrm{ml} / \mathrm{min}^{2}\right)$ & $145.7 \pm 5.0$ & $126.4 \pm 6.8$ & 0.025 \\
\hline$T_{30}$ & $33.9 \pm 4.2$ & $74.2 \pm 2.7$ & 0.028 \\
\hline
\end{tabular}

Data are mean $\pm S E$.

$D M$, diabetes mellitus; $H R$, heart rate; $S B P$, systolic blood pressure; $\dot{V} \mathrm{O}_{2}$, oxygen consumption; GER, gas exchange ratio; $\triangle H R$, change of $H R$ from rest to peak exercise; $\Delta O_{2}$ pulse, change of $\mathrm{O}_{2}$ pulse from rest to peak exercise; \%HRR, ratio of $\triangle H R$ to heart rate reserve estimated according to age and HRrest; T30, time constant of heart rate decay for the first $30 \mathrm{~s}$ of recovery period.

tical software program (SPSS Japan), and a p-value $<0.05$ was considered statistically significant.

\section{Results}

The patients' characteristics including age, body weight, height, body mass index, max CK-MB, HbA1c, LVEF, and location of AMI are shown in Table 2. There were no significant differences between the $\mathrm{DM}(+)$ and $\mathrm{DM}(-)$ groups in these parameters, except $\mathrm{HbA} 1 \mathrm{c}$, and medication for AMI. No significant difference was shown between the $\mathrm{DM}(+)$ and DM(-) groups in the proportion of multivessel disease evaluated by CAG performed on admission.

Table 3 shows the results obtained from CPX. No significant differences were shown between the DM(+) and $\mathrm{DM}(-)$ groups in HRAT, SBPAT, VंO2AT, GERAT and $\mathrm{O}_{2}$ pulseAT. There were also no significant differences between the 2 groups in exercise time, $\mathrm{SBP}_{\text {peak, }}$ GER peak, $\mathrm{O}_{2}$ pulsepeak and $\Delta \mathrm{O}_{2}$ pulse. However, HRpeak, $\mathrm{VO}_{2 \text { peak, }} \Delta \mathrm{HR}, \% \mathrm{HRR}$, HR slope, and $\dot{\mathrm{VO}}_{2}$ slope were significantly lower in the $\mathrm{DM}(+)$ group than in the $\mathrm{DM}(-)$ group $(\mathrm{p}=0.004, \mathrm{p}=0.002$, $\mathrm{p}<0.001, \mathrm{p}=0.002, \mathrm{p}=0.016$ and $\mathrm{p}=0.025$, respectively). T30 was significantly higher in the $\operatorname{DM}(+)$ group than in the $\mathrm{DM}(-)$ group $(\mathrm{p}=0.028)$.

Fig 3 shows the changes in LF, HF and LF/HF during CPX. Although there was no significant difference between the 2 groups in $\mathrm{LF} / \mathrm{HF}$ baseline, LFbaseline and $\mathrm{HFbaseline}$ were significantly lower in the $\mathrm{DM}(+)$ group than in the DM(-) group $(\mathrm{p}<0.01$ and $\mathrm{p}<0.01$, respectively). LF was significantly lower at HRrest $+20 \% \Delta \mathrm{HR}$ in the $\mathrm{DM}(+)$ group than in the DM $(-)$ group $(\mathrm{p}<0.01)$, and HF was also significantly lower at HRrest $+20 \% \Delta \mathrm{HR}$ and HRrest $+40 \% \Delta \mathrm{HR}$ in the $\mathrm{DM}(+)$ group $(\mathrm{p}<0.01$ and $\mathrm{p}<0.01$, respectively). There was no significant difference between the 2 groups in 

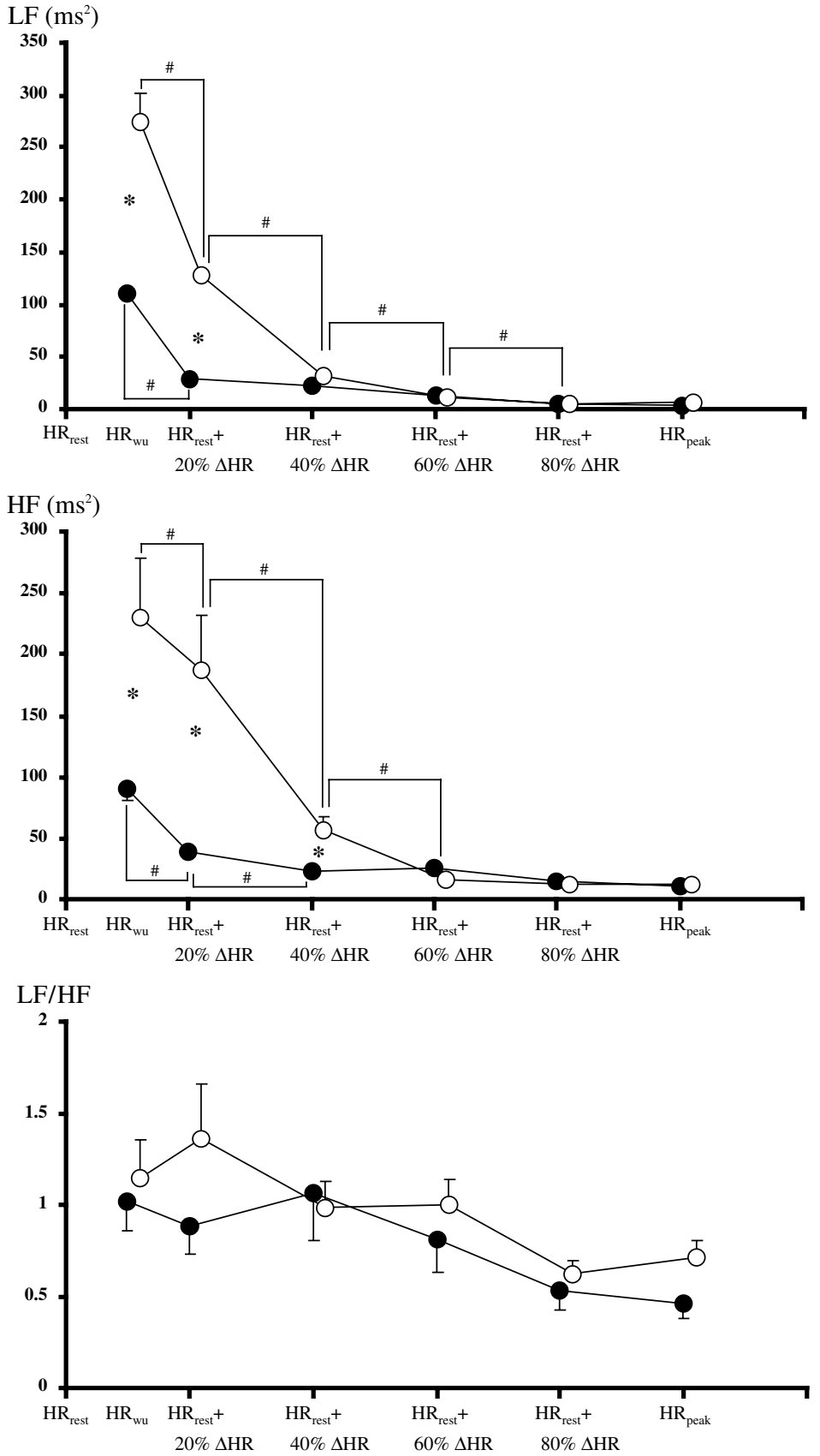

Fig 3. Changes in LF, HF and LF/HF during cardiopulmonary exercise testing. LF, low-frequency power in heart rate variability; $\mathrm{HF}$, high-frequency power in heart rate variability; $\mathrm{LF} / \mathrm{HF}$, the ratio of $\mathrm{LF}$ to $\mathrm{HF}$ in heart rate variability; $\Delta \mathrm{HR}$, heart rate (HR) change from $\mathrm{HR}$ at rest to $\mathrm{HR}$ at peak exercise; $\% \Delta \mathrm{HR}$, ratio to $\Delta \mathrm{HR}$. ( ) Diabetes mellitus $(\mathrm{DM})(+)$ group, $(\bigcirc) \mathrm{DM}(-)$ group. * Significant differencs between the 2 groups $(\mathrm{p}<0.01)$, \#significant difference between adjacent 2 stages $(\mathrm{p}<0.01)$.
LF/HF during CPX. Although LF and HF decreased significantly during CPX $(\mathrm{p}<0.01)$, they reached the minimal levels at lower $\mathrm{HR}$ in the $\mathrm{DM}(+)$ group as compared with the $\mathrm{DM}(-)$ group.

Table 4 shows the NE levels and HR response to exercise during CPX. Although there were no significant differences between the 2 groups in NEbaseline, NEpeak and $\log \Delta \mathrm{NE}$, $\Delta \mathrm{HR} / \log \Delta \mathrm{NE}$ and $\Delta \mathrm{HRHF}$ were significantly lower in the $\mathrm{DM}(+)$ group than in the $\mathrm{DM}(-)$ group $(\mathrm{p}<0.001$ and $\mathrm{p}<$ 0.001 , respectively).

Fig 4 shows the correlation between $\Delta \mathrm{HRHF}$ and $\Delta \mathrm{HR} / \log \Delta \mathrm{NE}$ in all 52 patients. There was a positive and significant correlation between $\Delta \mathrm{HRHF}$ and $\Delta \mathrm{HR} / \log \Delta \mathrm{NE}$ $(\mathrm{r}=0.63, \mathrm{p}<0.001)$.

Figs 5-7 show the correlations between $\Delta \mathrm{HR}$ and $\dot{\mathrm{V}} \mathrm{O}_{2 \text { peak, }}$ and between $\Delta \mathrm{HR} / \log \Delta \mathrm{NE}$ or $\Delta \mathrm{HRHF}$ and $\dot{\mathrm{V}} \mathrm{O}_{2 \text { peak }}$ in all 52
Table 4 NE Levels and HR Response to Exercise

\begin{tabular}{lccc}
\hline \hline & $\begin{array}{c}\text { DM(-) } \\
\text { group }\end{array}$ & $\begin{array}{c}\text { DM }(+) \\
\text { group }\end{array}$ & p value \\
\hline NEbaseline $(\mathrm{pg} / \mathrm{ml})$ & $239.2 \pm 17.2$ & $221.0 \pm 29.1$ & $\mathrm{NS}$ \\
$N E_{\text {peak }}(\mathrm{pg} / \mathrm{ml})$ & $1,933.6 \pm 182.3$ & $1,640.0 \pm 206.5$ & $\mathrm{NS}$ \\
$\log \Delta N E$ & $7.3 \pm 0.1$ & $7.1 \pm 0.1$ & $\mathrm{NS}$ \\
$\Delta$ HR/log $\Delta N E$ & $10.6 \pm 0.4$ & $8.3 \pm 0.3$ & $<0.001$ \\
$\Delta H R H F($ beats $/$ min $)$ & $29.4 \pm 1.7$ & $16.4 \pm 1.4$ & $<0.001$ \\
\hline
\end{tabular}

Data are mean $\pm S E$.

$N E$, norepinephrine; HR, heart rate; DM, diabetes mellitus; $\triangle N E$, change of $N E$ concentration from rest to peak exercise; $\Delta H R$, change of $H R$ from rest to peak exercise; $\triangle H R H F, H R$ change from $H R$ at warm-up to $H R$ at the minimum level during cardiopulmonary exercise testing. 


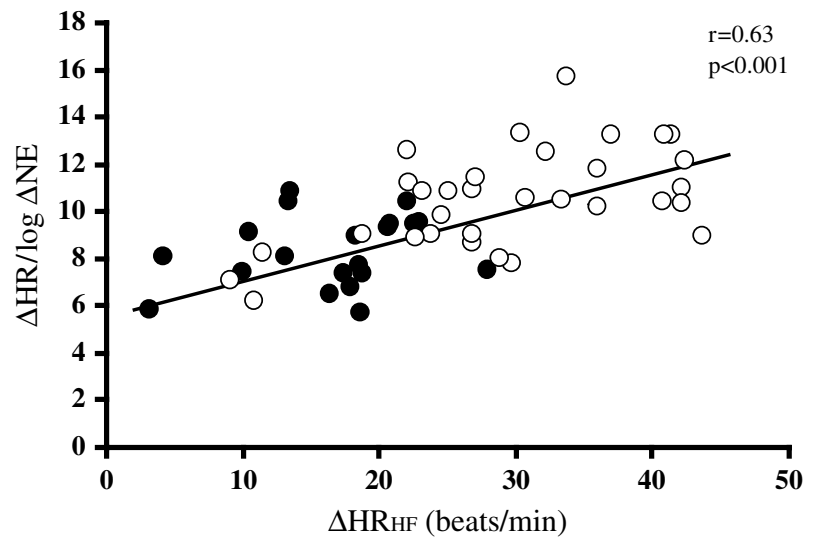

Fig 4. Correlation between $\Delta \mathrm{HRHF}$ and $\Delta \mathrm{HR} / \log \Delta \mathrm{NE}$. $\Delta \mathrm{HRHF}$, heart rate (HR) change from HR at warm-up to the HR when $\mathrm{HF}$ reached the minimum level; $\Delta \mathrm{HR}, \mathrm{HR}$ change from $\mathrm{HR}$ at rest to HR at peak exercise; $\triangle \mathrm{NE}$, norepinephrine (NE) change from $\mathrm{NE}$ at rest to $\mathrm{NE}$ at peak exercise. (๑) Diabetes mellitus $(\mathrm{DM})(+)$ group, $(\bigcirc) \mathrm{DM}(-)$ group.

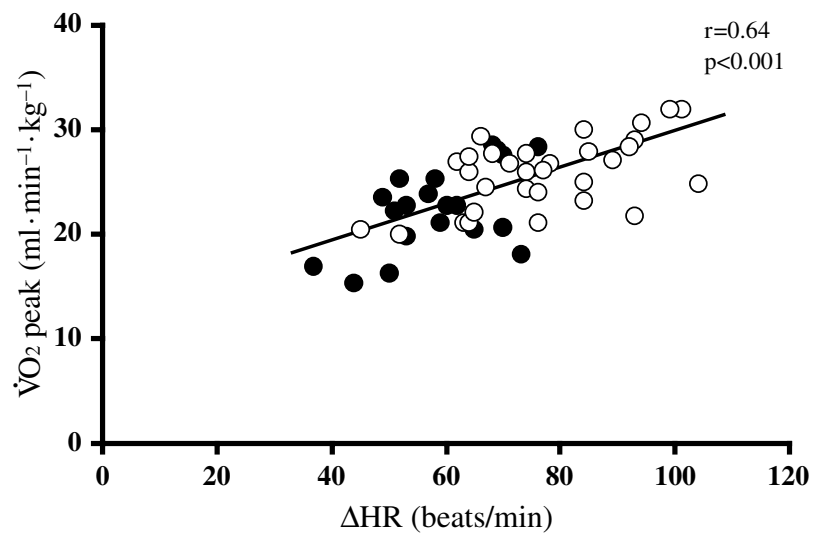

Fig 5. Correlation between $\Delta \mathrm{HR}$ and $\dot{\mathrm{V}} \mathrm{O}_{2 \text { peak. }} \Delta \mathrm{HR}$, heart rate (HR) change from $\mathrm{HR}$ at rest to $\mathrm{HR}$ at peak exercise; $\dot{\mathrm{V}}$ 2peak, oxygen consumption at peak exercise. $(\bullet)$ Diabetes mellitus $(\mathrm{DM})(+)$ group, (○) DM(-) group.

patients. There were positive correlations between $\triangle \mathrm{HR}$ and $\dot{\mathrm{V}} \mathrm{O}_{2 \text { peak }}(\mathrm{r}=0.64, \mathrm{p}<0.001)$, between $\Delta \mathrm{HR} / \log \Delta \mathrm{NE}$ and $\dot{\mathrm{V}} \mathrm{O}_{2 \text { peak, }}(\mathrm{r}=0.63, \mathrm{p}<0.001)$, and between $\Delta \mathrm{HRHF}$ and $\dot{\mathrm{V}} \mathrm{O}_{2 \text { peak }}(\mathrm{r}=0.51, \mathrm{p}<0.001)$. However, there were no significant correlations between LVEF, max CK-MB, NEbaseline or $\mathrm{HFbaseline}$, and $\dot{\mathrm{V} O}$ 2peak $(\mathrm{p}=0.476, \mathrm{p}=0.421, \mathrm{p}=0.089$, and $\mathrm{p}=0.060$, respectively).

Fig 8 shows the comparison between HRHF and HRAT in each group. There was no significant difference between HRAT and HRHF in the DM(-) group ( $\mathrm{p}=0.254)$, but, in contrast, HRHF was significantly lower than HRAT in the $\mathrm{DM}(+)$ group $(\mathrm{p}=0.005)$.

Fig 9 shows the ratio of HRAT or HRHF to HRpeak in the 2 groups. The ratio of HRAT to HRpeak was significantly higher in the $\mathrm{DM}(+)$ group than in the $\mathrm{DM}(-)$ group $(\mathrm{p}=0.004)$, whereas there was no significant difference in the ratio of HRHF to HRpeak between the 2 groups.

\section{Discussion}

In the present study, we focused on the HR response to exercise, which is derived from the balance between SN

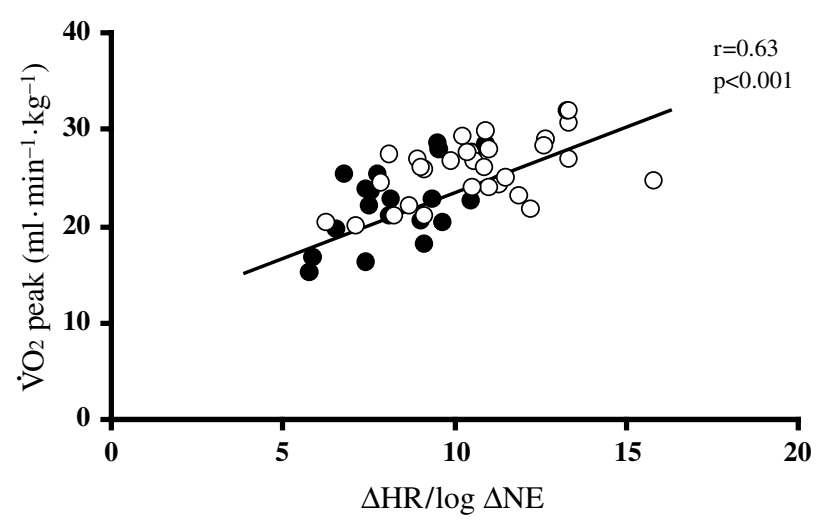

Fig 6. Correlation between $\Delta \mathrm{HR} / \log \Delta \mathrm{NE}$ and $\dot{\mathrm{V}} \mathrm{O}_{2 \text { peak. }} \Delta \mathrm{HR}$, heart rate (HR) change from $\mathrm{HR}$ at rest to $\mathrm{HR}$ at peak exercise; $\Delta \mathrm{NE}$, norepinephrine (NE) change from $\mathrm{NE}$ at rest to $\mathrm{NE}$ at peak exercise; $\dot{\mathrm{V} O}$ 2peak, oxygen consumption at peak exercise. (๑) Diabetes mellitus $(\mathrm{DM})(+)$ group, $(\bigcirc) \mathrm{DM}(-)$ group.

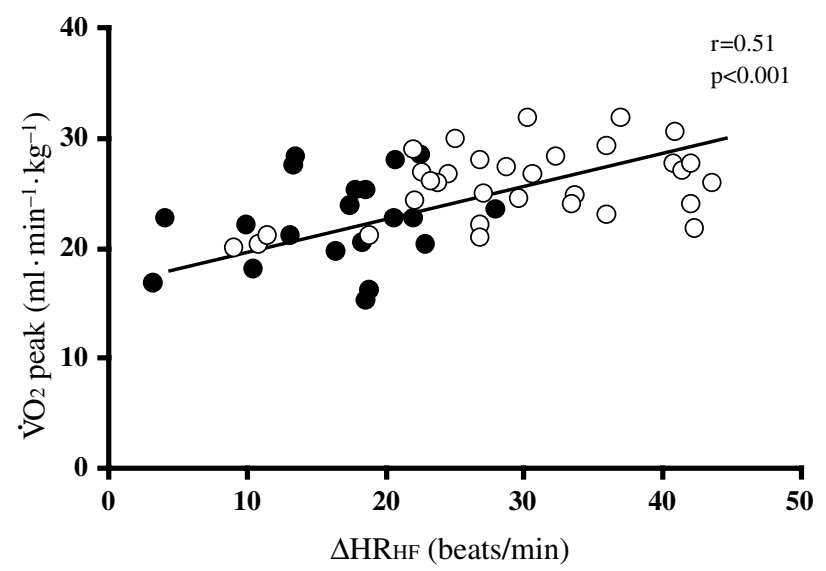

Fig 7. Correlation between $\Delta \mathrm{HRHF}$ and $\dot{\mathrm{V}} \mathrm{O}_{2 \text { peak. }} \Delta \mathrm{HRHF}$, heart rate (HR) change from HR at warm-up to the HR when high-frequency power (HF) reached the minimum level; $\dot{\mathrm{V} O}$ 2peak, oxygen consumption at peak exercise. $(\bullet)$ Diabetes mellitus $(\mathrm{DM})(+)$ group, $(\bigcirc)$ $\mathrm{DM}(-)$ group.

and PN activities, during incremental exercise in patients with DM who were regaining health after AMI. Previous studies report that AMI patients with DM have a lower exercise capacity than those without DM, which possibly results from inappropriate secretion of catecholamines in the heart 3,7 In addition, studies using myocardial scintigraphy have shown a positive correlation between ${ }^{123}$ I-metaiodobenzylguanidine uptake and peak HR during incremental exercise in patients with congestive heart failure, indicating that decreased secretion of NE in the sinus node affected the HR response to exercise 23 Bottini et al reported that the elevation of serum catecholamine during incremental exercise was lower in patients with DM suffering from autonomic nervous neuropathy than in normal subjects? and noted that SN dysfunction caused an inadequate HR response to exercise in DM patients, inducing an insecure cardiocirculatory condition? ${ }^{4}$

As the HR response corresponding to the increase of serum NE is less sufficient in patients with congestive heart failure than in normal subjects, it is also suggested that the sensitivity of the sinus node to NE has deteriorated in these patients 25 The present study showed that severe SN dys- 


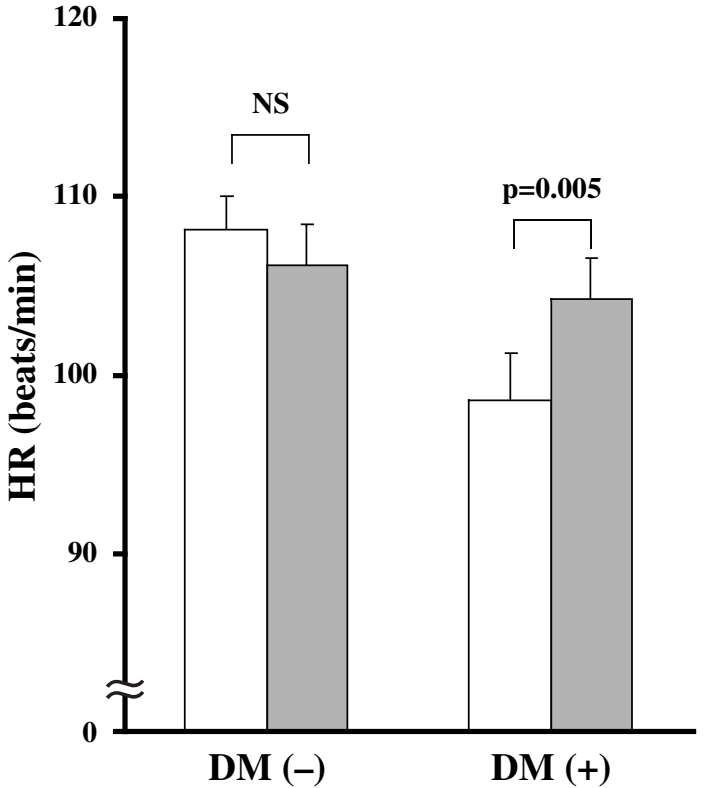

Fig 8. Comparison of between HRHF and HRAT in each group. Diabetes mellitus $(\mathrm{DM})(-)$, patients without diabetes mellitus; $\mathrm{DM}(+)$, patients with diabetes mellitus; HR, heart rate. Open bars indicate the HR when the high-frequency power reached the minimum level (HRHF), Closed bars indicate the HR at the anaerobic threshold.

function was not observed, even in the $\mathrm{DM}(+)$ group, because there were no significant differences between the $\mathrm{DM}(+)$ and $\mathrm{DM}(-)$ groups in NEbaseline, LF/HFbaseline, $\mathrm{LF} / \mathrm{HF}_{\text {peak }}$ and NEpeak as indicators of SN activity. Furthermore, the study showed a close and positive correlation between $\Delta \mathrm{HR} / \log \Delta \mathrm{NE}$ and $\dot{\mathrm{V}}_{2}$ peak and a significantly lower value of $\Delta \mathrm{HR} / \log \Delta \mathrm{NE}$ in the $\mathrm{DM}(+)$ group as compared with the DM(-) group, indicating that the sensitivity of the sinus node to NE plays a more important role in the elevation of HR than does the amount of NE secreted from the $\mathrm{SN}$ terminal. Therefore, it seems that an inadequate HR response to exercise is a major factor in the decline in exercise capacity of patients with AMI and DM, and that $\Delta \mathrm{HR} / \log \Delta \mathrm{NE}$, reflecting the HR response derived from $\mathrm{SN}$ activity, may be an useful indicator in the assessment of the exercise capacity of AMI patients.

It was previously reported that PN activity at rest assessed by HRV shows a positive correlation with maximal $\dot{\mathrm{V}} \mathrm{O}_{2}$ in healthy subjects, leading to the conclusion that the decrease in PN activity associated with aging is related to the decline in exercise capacity that is also associated with aging 26 However, there are few reports of the relationship between the HR response derived from PN activity and the exercise capacity of AMI patients with DM, although DM patients are known to show lower PN activity compared with normal subjects? The HF in HRV is commonly used to estimate the change inf PN activity, because it decreases in response to increasing exercise intensity or inhibition of cholinergic receptors during exercise ${ }^{27}$ In the present study, we assessed HRV using the MemCalc method, which can analyze the power spectral densities of a time series $20 \mathrm{We}$ found that $\mathrm{HF}$ was significantly lower during exercise and reached the minimum level at a lower HR in the DM(+) group as compared with the $\mathrm{DM}(-)$ group, indicating that $\mathrm{PN}$ dysfunction shown by a significant decrease of $\Delta \mathrm{HRHF}$

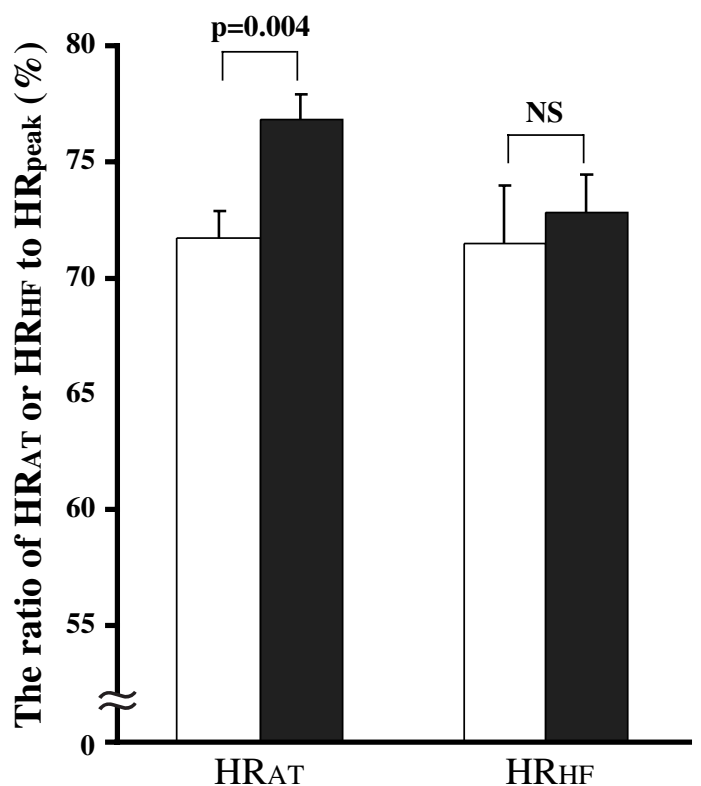

Fig 9. Comparisons in the ratio of HRAT or HRHF to HRpeak between the 2 groups. HRHF, heart rate (HR) when the high-frequency power reached the minimum level; HRAT, HR at the anaerobic threshold. Open bars indicate the Diabetes mellitus (DM) (-)group, Closed bars indicate the $\mathrm{DM}(+)$ group.

caused an inadequate HR response to exercise. In addition, it seemed that the decline in exercise capacity resulted from an inadequate HR response to exercise in AMI patients with DM, because there was a positive correlation between $\triangle$ HRHF and $\dot{\mathrm{V}}$ 2peak.

It is well known that cardiopulmonary function, blood flow to skeletal muscles, and muscle oxygen usage are important factors, together with age and sex, in regulating exercise capacity. An inadequate increase in stroke volume is one reason for the decline in exercise capacity of patients with AMI and DM, particularly those who have diabetic cardiomyopathy or lower LVEF because of severe AMI 28 The present study, however, showed no significant differences in LVEF, $\mathrm{O}_{2}$ pulsepeak and $\Delta \mathrm{O}_{2}$ pulse between the 2 groups. Because the DM(+) group consisted of patients with AMI who had mild to moderate cardiac dysfunction and could perform the exercise training using a treadmill or cycle ergometer, the present study failed to show that left ventricular function was one of the main factors causing the decline in exercise capacity. However, we assessed the $\mathrm{O}_{2}$ pulse to estimate the stroke volume during exercise. A previous study reported that the $\mathrm{O}_{2}$ pulse was equivalent to the stroke volume calculated echocardiographically at the maximal HR during ramp exercise ${ }^{8}$ Therefore, we believe that the $\mathrm{O}_{2}$ pulse substantially reflects the stroke volume and is a parameter of cardiac function during exercise. It is generally known that HR is 2- to 3-fold higher at maximum exercise than at rest, despite only 1.5- to 2-fold increase in stroke volume ${ }^{29}$ Although the present study showed a positive correlation between $\Delta \mathrm{HR}$ and $\dot{\mathrm{V}} \mathrm{O}_{2 \text { peak }}$ in all patients, both $\triangle \mathrm{HR}$ and HR slope were significantly lower in the $\mathrm{DM}(+)$ group as compared with those in the $\mathrm{DM}(-)$ group. Therefore, it seems that the inadequate increase in cardiac output during exercise is caused by poorer elevation of HR than of stroke volume in AMI patients with DM, if they 
have the same resting cardiac function as patients without DM.

As regards DM treatment, a previous study reported that insulin injection decreased $\mathrm{HF}$ and increased simultaneously $\mathrm{LF} / \mathrm{HF}$, as assessed by HRV in healthy subjects, resulting in an increment of HR at rest $3^{0}$ However, there is also a study reporting no significant changes of $\mathrm{HF}$ and $\mathrm{LF} / \mathrm{HF}$ after an injection of insulin to patients with DM. The present study in which 7 patients in the DM(+) group had insulin therapy showed no significant differences between the 2 groups in $\mathrm{NE}$ at rest and peak exercise, and in HR at rest. Therefore, we consider that the difference in treatment regimens for DM had less influence on the study results in the comparison to the HR response. Furthermore, poor control of blood glucose is generally known to change the autonomic nervous activities regulating $\mathrm{HR}$ response to exercise in patients with DM. Larsen et al reported that the peak HR during incremental exercise was lower in the patients with poorly controlled DM $\left(\mathrm{HbAlc}_{1 \mathrm{c}}>8.4 \%\right)$ than in those who were well controlled ${ }^{1}$ In the present study, the mean $\mathrm{HbA} 1 \mathrm{c}$ was $7.2 \pm 0.2 \%$ in the $\mathrm{DM}(+)$ group and none had $\mathrm{HbA} 1 \mathrm{c}$ $>8.0 \%$, indicating poor control of DM 32 The patients with $\mathrm{DM}$ in the present study maintained a satisfactory level of blood glucose after admission to hospital. However, the $\mathrm{DM}(+)$ group showed significantly lower HRpeak and $\Delta \mathrm{HR}$ than the DM(-) group, suggesting that AMI patients with DM had an insufficient HR response to exercise despite appropriate control of blood glucose, compared with those without DM.

The maximum $\dot{\mathrm{V}} \mathrm{O}_{2}$ or maximum HR obtained from CPX is often used as the target HR in the prescription of exercise therapy 33 However, the value assessed by the maximum exercise should not be the target HR for patients in the acute phase of AMI, because it is difficult for them to perform at that level. Therefore, the AT obtained from the submaximum exercise is used for them to carry out exercise training at the target intensity. It is well known that the regular exercise training at the AT improves exercise capacity without a remarkable elevation of SN activity and serum lactic acid level!2,13,34 The present study showed no significant difference between HRHF and HRAT in the $\mathrm{DM}(-)$ group, supporting the previous report that PN activity reaches the lowest value at approximately $110 \%$ of AT in incremental exercise testing! ${ }^{13}$ However, HRAT was significantly higher than $\mathrm{HRHF}$ in the $\mathrm{DM}(+)$ group, and the ratio of HRAT to HRpeak was also higher in the DM(+) group than in the $\mathrm{DM}(-)$ group. In addition, a positive correlation between $\Delta \mathrm{HRHF}$ and $\Delta \mathrm{HR} / \log \Delta \mathrm{NE}$ shown in the present study indicates that $\mathrm{SN}$ activity was elevated more excessively to obtain an adequate HR increase in AMI patients who showed an inadequate HR increase derived from PN dysfunction. These findings suggest that in the DM(+) group $\mathrm{SN}$ activity is more dominant in the regulation of HR at the intensity of the AT than is PN activity, and that the relative exercise intensity is stronger in the $\mathrm{DM}(+)$ group as compared with the DM(-) group. Therefore, the present study indicates that the more exercise intensity is loaded toward the AT, the more activation of $\mathrm{SN}$ is induced in the $\mathrm{DM}(+)$ group. Continuing the exercise at the intensity near AT may induce a hyperglycemic state during exercise in AMI patients with DM 35 In addition, we should beware that the elevated SN activity causes excessive blood pressure elevation or exercise-induced arrhythmias in such patients, even at exercise training intensities lower than the AT.

\section{Study Limitations}

In the present study, we failed to evaluate the serial change of SN activity by using the LF/HF in HRV because of its dispersion, and we measured serum catecholamine levels at only 2 points (ie, rest and peak exercise). Blood samples need to be collected at various points during incremental exercise in future studies to detect the point of SN activation during exercise. Because the effects of regular exercise training on SN and PN activities is still unclear in the present study, further studies are needed to investigate the HR response to exercise derived from autonomic nervous function after regular exercise.

In the present study, various medical treatments were given to the patients with DM. Because there was an ethical difficulty in giving uniform therapy to them, we performed the study without modifying the treatments for DM. Therefore, in the future we should analyze the HR response to exercise among subgroups classified according to the DM treatment regimen.

\section{Conclusions}

The insufficient HR response to exercise derived from both of SN and PN dysfunction is a significant factor in the decreased exercise capacity of patients with AMI complicated with DM. The HR response to exercise is affected by $\mathrm{SN}$ activity, even at exercise intensities below the AT, in patients with AMI and DM, although it is commonly regulated by PN activity at intensities up to the AT in those without DM.

\section{Acknowledgment}

This study was supported in part by a grant-in-aid for scientific research (Grant no. 15500383) from the Ministry of Education, Culture, Sports, Science and Technology in Japan.

\section{References}

1. Yu CM, Lau CP, Cheung BMY, Fong YM, Ho YY, Lam KB, et al. Clinical predictors of morbidity and mortality in patients with myocardial infarction or revascularization who underwent cardiac rehabilitation, and importance of diabetes mellitus and exercise capacity. Am J Cardiol 2000; 85: 344-349.

2. Fujishima M, Kiyohara Y, Kato I, Ohmura T, Iwamoto H, Nakayama $\mathrm{K}$, et al. Diabetes and cardiovascular disease in a prospective populations survey in Japan: The Hisayama study. Diabetes 1996; 45: S14S16.

3. Izawa K, Tanabe K, Omiya K, Yamada S, Yokoyama Y, Ishiguro T, et al. Impaired chronotropic response to exercise in acute myocardial infarction patients with type 2 diabetes mellitus. Jpn Heart J 2003; 44: $187-199$.

4. Izawa K, Hirano Y, Yamada S, Oka K, Omiya K, Iijima S. Improvement in physiological outcomes and health-related quality of life following cardiac rehabilitation in patients with acute myocardial infarction. Circ J 2004; 68: 315-320.

5. Robinson B, Epstein SE, Beiser GD, Braunwald E. Control of heart rate by the autonomic nervous system. Circ Res 1966; 29: 400-411.

6. Kremser CB, Levitt NS, Borow KM, Jaspan JB, Lindbloom C, Polonsky KS, et al. Oxygen uptake kinetics during exercise in diabetic neuropathy. J Appl Physiol 1988; 65: 2665-2671.

7. Bottini P, Tantucci C, Scionti L, Dottorini ML, Puxeddu E, Reboldi $\mathrm{G}$, et al. Cardiovascular response to exercise in diabetic patients: Influence of autonomic neuropathy of different severity. Diabetologia 1995; 38: $244-250$.

8. Bernardi L, Ricordi L, Lazzari P, Solda P, Calciati A, Ferrari MR, et al. Impaired circadian modulation of sympathovagal activity in diabetes: A possible explanation for altered temporal onset of cardiovascular disease. Circulation 1992; 86: 1443-1452.

9. Matsunaga A, Masuda T, Ogura MN, Saitoh M, Kasahara Y, Iwamura $\mathrm{T}$, et al. Adaptation to low-intensity exercise on a cycle ergometer by patients with acute myocardial infarction undergoing phase 1 cardiac rehabilitation. Circ J 2004; 68: 938-945. 
10. Miyakoda H, Noguchi N, Matsumoto T, Kitamura H, Kinugawa T, Ogino K, et al. Plasma catecholamine responses to dynamic exercise in patients with coronary artery disease: The relationship between sympathetic activity and systolic blood pressure and exercise-induced ventricular arrhythmias. Jpn Circ J 1992; 56: 1115-1123.

11. Beaver WL, Wasserman K, Whipp BJ. A new method for detecting anaerobic threshold by gas exchange. J Appl Physiol 1988; 60: $2020-2027$.

12. Ivy JL, Withers RT, Van Handel PJ, Elger DH, Cosrill DL. Muscle respiratory capacity and fiber type as determinants of the lactate threshold. J Appl Physiol 1980; 48: 523-527.

13. Yamamoto Y, Hughson RL, Nakamura Y. Autonomic nervous system responses to exercise in relation to ventilatory threshold. Chest 1992; 101(Suppl): 206s-210s.

14. Bruce RA, Kusumi F, Hosmer D. Maximal oxygen uptake and nomographic assessment of functional aerobic impairment in cardiovascular disease. Am Heart J 1973; 85: 546-562.

15. American College of Sports Medicine. Clinical exercise testing. In Flanklin BA, editor. ACSM's guidelines for exercise testing and prescription, $6^{\text {th }}$ edn. Baltimore: Williams \& Wilkins; 2000; $91-114$.

16. Imai K, Sato H, Hori M, Kusuoka H, Ozaki H, Yokoyama H, et al. Vagally mediated heart rate recovery after exercise is accelerated in athletes but blunted in patients with chronic heart failure. $\mathrm{J} \mathrm{Am} \mathrm{Coll}$ Cardiol 1994; 24: 1529-1535.

17. Pashlow FJ, Dafoe WA. Clinical cardiac rehabilitation: A cardiologist's guide, $2^{\text {nd }}$ ed. Maryland: Williams \& Wilkins; 1999; $102-120$.

18. Sundstedt M, Hedberg P, Jonason T, Ringqvist I, Brodin LA, Henriksen E. Left ventricular volumes during exercise in endurance athletes assessed by achocardiography. Acta Physiol Scand 2004; 182: $45-51$.

19. Kanaya S, Fujino T, Komiya S, Ogaki T, Komuro T, Suzuki S, et al. The responses of plasma catecholamines, serum potassium, lipids and blood glucose levels during graded exercise and recovery. Kenkokagaku 1985; 7: 51-59.

20. Ohtomo N, Kamo T, Watanabe M, Yoneyama K, Tanaka Y, Hayashi R. Power spectral densities of temporal variations of brood pressures. Jpn J Appl Physiol 1996; 35: 5571-5582.

21. Moritani T, Hayashi T, Shinohara M, Mimasa F, Shibata M. Comparison of sympatho-vagal function among diabetic patients, normal controls and endurance athletes by heart rate spectral analysis. $J$ Sports Med Sci 1993; 7: 31-39.

22. Shibata M, Moritani T, Miyawaki T, Hayashi T, Nakao K. Exercise prescription based upon cardiac vagal activity for middle-aged obese women. Int J Obes 2002; 26: 1356-1362.
23. Sato M, Maehara K, Yaoita H, Otani H, Hirosaka A, Saito T, et al. Correlation between cardiac norepinephrine overflow during exercise and cardiac 123I-MIBG uptake in patients with chronic heart failure. J Nucl Med 2003; 44: 1618-1624.

24. Hilsted J, Galbo H, Christensen NJ, Parving H-H, Benn J. Hemodynamic changes during graded exercise in patients with diabetic autonomic neuropathy. Diabetes Care 1982; 22: 318-323.

25. Colucci WS, Ribeiro JP, Rocco MB, Quigg RJ, Creager MA, Marsh $\mathrm{JD}$, et al. Impaired chronotropic response to exercise in patients with congestive heart failure: Role of postsynaptic beta-adrenergic desensitization. Circulation 1989; 80: 314-323.

26. Goldsmith RL, Bigger JT Jr, Bloomfield DM, Steinman RC. Physical fitness as a determinant of vagal modulation. Med Sci Sports Exerc 1997; 29: 812-817.

27. Warren JH, Jaffe RS, Wraa CE, Stebbins CL. Effect of autonomic blockade on power spectrum of heart rate variability during exercise. Am J Physiol 1997; 273: R495-R502.

28. Orlander PR, Goff DC, Morrissey M, Ramsey DJ, Wear ML, Labarthe DR, et al. The relation of diabetes to the severity of acute myocardial infarction and post-myocardial infarction survival in Mexican-Americans and non-Hispanic whites: The Corpus Christi Heart Project. Diabetes 1994; 43: 897-902.

29. Astrand PO, Cuddy TE, Saltin B, Stenberg J. Cardiac output during submaximal and maximal work. J Appl Physiol 1964; 19: 268-274.

30. Paolisso G, Manzella D, Rizzo MR, Barbieri M, Varrricchio G, Gambardella A, et al. Effects of insulin of the cardiac autonomic nervous system in insulin-resistant states. Clin Sci 2000; 98: 129136.

31. Larsen JR, Sjoholm H, Berg TJ, Sandvik L, Brekke M, Hannsen KF, et al. Eighteen years of fair glycemic control preserves cardiac autonomic function in type 1 diabetes. Diabetes Care 2004; 27: $963-$ 966.

32. Mazze RS, Strock E, Simonson G, Bergenstal R, Eztwiler D. Staged diabetes management, a systematic approach. Minneapolis, MN: International Diabetes Center, 2000; 20-21.

33. Fletcher GF, Balady GJ, Amsterdam EA, Chaitman B, Eckel R, Fleg $\mathrm{J}$, et al. Exercise standards for testing and training: A statement for healthcare professionals from American Heart Association. Circulation 2001; 104: 1694-1740.

34. The Japanese Circulation Society. Guidelines for exercise training in patients with heart disease (JCS 2002). Circ J 2002; 66(Suppl 4): $1177-1247$.

35. Wahren J, Felig P, Hagenfeldt L. Physical exercise and fuel homeostasis in diabetes mellitus. Diabetologia 1978; 14: 213-222. 